TITLE:

\title{
Mechanism of photoluminescence enhancement in single semiconductor nanocrystals on metal surfaces
}

\section{AUTHOR(S):}

Ito, Yuichi; Matsuda, Kazunari; Kanemitsu, Yoshihiko

\section{CITATION:}

Ito, Yuichi ...[et al]. Mechanism of photoluminescence enhancement in single

semiconductor nanocrystals on metal surfaces. Physical Review B 2007, 75(3): 033309.

ISSUE DATE:

2007-01

URL:

http://hdl.handle.net/2433/87358

RIGHT:

c 2007 The American Physical Society 


\title{
Mechanism of photoluminescence enhancement in single semiconductor nanocrystals on metal surfaces
}

\author{
Yuichi Ito, Kazunari Matsuda, and Yoshihiko Kanemitsu* \\ Institute for Chemical Research, Kyoto University, Gokasho, Uji, Kyoto 611-0011, Japan
}

(Received 22 September 2006; published 17 January 2007)

\begin{abstract}
We have studied the mechanism of the photoluminescence (PL) enhancement and quenching of single $\mathrm{CdSe} / \mathrm{ZnS}$ core/shell nanocrystals on Au surfaces by means of single nanocrystal spectroscopy. The on-off PL blinking observed on the glass surface is drastically suppressed on Au surfaces, because of the fast energy transfer between Au surfaces and nanocrystals. The PL enhancement of single CdSe/ZnS nanocrystals occurs on rough $\mathrm{Au}$ surfaces, but PL quenching occurs on flat Au surfaces, compared to the case of the glass surface. Single nanocrystal spectroscopy reveals that the PL enhancement on rough Au surfaces is caused by the suppression of PL blinking and the electric field enhancement due to localized plasmon excitation.
\end{abstract}

DOI: 10.1103/PhysRevB.75.033309

PACS number(s): 78.67.Bf, 73.21.-b

Colloidal semiconductor nanocrystals with high photoluminescence (PL) quantum efficiencies have been extensively studied both from the viewpoint of fundamental physics and with consideration for the potential applications to electronics and biotechnology. Single nanocrystal spectroscopy is one of the most useful tools to reveal the intrinsic nature of nanocrystals hidden by sample inhomogeneity, such as size fluctuations and surface variations in macroscopic ensembleaveraged experiments. ${ }^{1-3}$ Many unique and inherent optical phenomena have been discovered by means of single nanocrystal spectroscopy; very narrow spectral linewidth, ${ }^{1}$ single photon antibunching, ${ }^{2}$ and PL blinking. ${ }^{3}$ PL blinking is attributed to a random switching between emitting "on" and nonemitting "off" states even under continuous-wave laser excitation. This PL blinking behavior of single nanocrystals is very sensitive to the immediate environments surrounding nanocrystals. ${ }^{4-14}$ Therefore, the measurement of PL blinking becomes a good probe for the ongoing discussion on the energy transfer in hetero-nanostructures, such as nanocrystalmetal complexes. The interfaces between metals and nanocrystals play complex and essential roles in the optical responses of semiconductor nanocrystals on metals: PL enhancement and/or quenching occurs on metal surfaces. ${ }^{14-18}$ The PL mechanism of chromophore molecules and nanocrystals on metal surfaces is not clear and is still under discussion. The detailed understanding interactions between nanocrystals and metal surface are very important to enhance the PL intensity of nanocrystals and to decrease the $\mathrm{PL}$ off times in conjunction with the improvement of the PL efficiency of nanocrystals.

In this paper, we report a drastic change of the PL intensity and time-trace of single $\mathrm{CdSe} / \mathrm{ZnS}$ nanocrystals on $\mathrm{Au}$ surfaces relative to those on glass surfaces at room temperature. The PL blinking of a single $\mathrm{CdSe} / \mathrm{ZnS}$ nanocrystal is suppressed on both rough and flat Au surfaces. Under resonance excitation of the localized plasmons, the PL intensity on the rough $\mathrm{Au}$ surface is one order of magnitude more than that on the glass surface. The PL enhancement on rough $\mathrm{Au}$ surfaces is caused by the electric field enhancement due to localized plasmon excitation and the suppression of PL blinking.

The averaged core size of the $\mathrm{CdSe} / \mathrm{ZnS}$ core/shell nano- crystals (Evident Technologies Corporation) used in this work was $5.2 \mathrm{~nm}$, and their PL wavelength at room temperature was $620 \mathrm{~nm}$. Two types of Au films with flat and rough surfaces have been prepared on glass substrates. The roughsurface $\mathrm{Au}$ films were fabricated by an $\mathrm{Ar}^{+}$sputtering method. The flat-surface Au films were grown by an electron beam evaporation method; $\mathrm{Cr}$ thin films with thickness of $5 \mathrm{~nm}$ were first grown as a buffer layer on the glass substrates, and the $100 \mathrm{~nm}$-thick Au films were then evaporated in a $10^{-9}$ Torr atmosphere. Figures $1(\mathrm{a})$ and $1(\mathrm{~b})$ show the atomic force microscopy (AFM) images of rough and flat $\mathrm{Au}$ surfaces on the same vertical scales. The rough Au surfaces are composed of the features with peaks and valleys of 20-50 nm sizes. On the other hand, the root mean squared (RMS) value of the topography on the flat Au surfaces was estimated to be less than $1 \mathrm{~nm}$. CdSe/ZnS nanocrystals were dispersed on cover glasses or $\mathrm{Au}$ films by a spin-coating technique.

Single nanocrystal PL measurements were carried out at room temperature using a home-built scanning confocal microscope. The $\mathrm{CdSe} / \mathrm{ZnS}$ core/shell nanocrystal samples on different substrates were excited with a continuous-wave $\mathrm{Ar}^{+}$ laser $(457.9,488$, and $514.5 \mathrm{~nm})$, a $\mathrm{Nd}^{3+}: \mathrm{YVO}_{4}$ laser $(532 \mathrm{~nm})$, and a He-Ne laser $(594 \mathrm{~nm})$. The laser light was focused on the sample surface through a microscope objective (NA 0.8). The excitation power density was kept typically below $300 \mathrm{~W} / \mathrm{cm}^{2}$. The PL signals were detected by a single photon-counting $\mathrm{Si}$ avalanche photodiode through a band-pass filter with a center wavelength of $630 \mathrm{~nm}$ and a
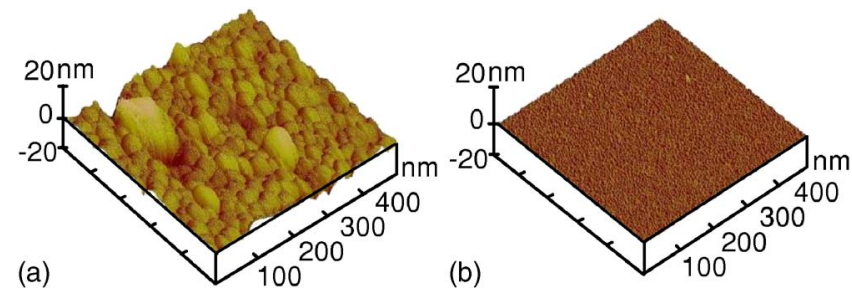

FIG. 1. (Color online) (a) and (b) Topographic images of rough (left side) and flat (right side) Au surfaces measured using an atomic force microscope (AFM). The vertical axes of both images are drawn using the same scale. 

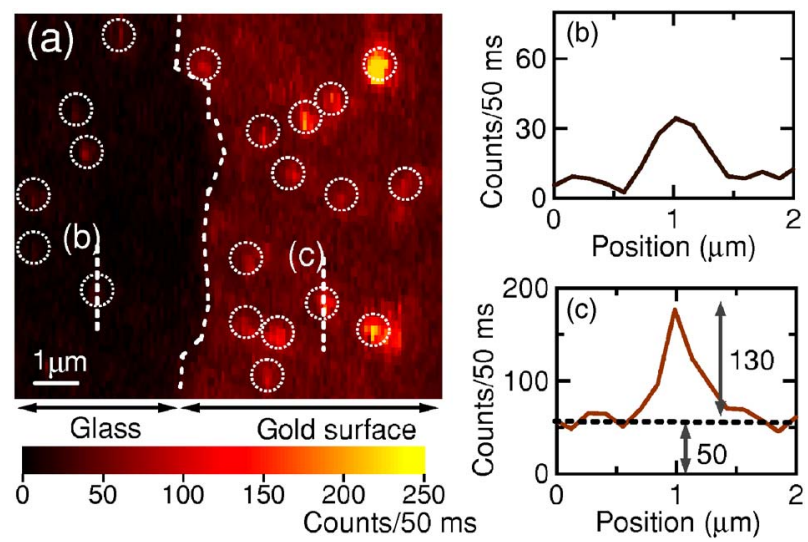

FIG. 2. (Color online) (a) PL image of single CdSe/ZnS nanocrystals on a rough Au surface at room temperature. A dotted line marks the border between the glass (left side of the image) and rough (right side) Au surfaces. (b) and (c) Cross-sectional PL intensity profiles obtained from the PL images.

bandwidth of $30 \mathrm{~nm}$. The detector accumulation time was $50 \mathrm{~ms}$ on the measurements of the PL intensity time-trace.

Figure 2(a) shows a PL image of single $\mathrm{CdSe} / \mathrm{ZnS}$ nanocrystals on a rough Au surface under $488 \mathrm{~nm}$ excitation at room temperature. Both the glass and rough Au surface regions were prepared on the same substrate. The dotted line in Fig. 2(a) shows a boarder between the glass surface (the left side) and the rough Au surface (the right side). Each bright spot on the rough $\mathrm{Au}$ and the glass surface [dotted circles in Fig. 2(a)] comes from the PL from single CdSe/ZnS nanocrystals. The difference in the single-nanocrystal PL intensity between the glass and rough Au surface regions can be clearly observed. The cross-sectional PL intensity profiles on the glass and rough Au surfaces are shown in Figs. 2(b) and 2(c), respectively. The nominal PL intensity after the subtraction of the background signals on the rough Au surfaces is close to 130 counts $/ 50 \mathrm{~ms}$, and this intensity is larger than that on the glass surface $(\sim 30$ counts $/ 50 \mathrm{~ms})$. The $4-5$ time enhancement of the PL intensity can be typically observed on rough-surface Au substrates.

Figure 3(a) shows the PL intensity time-trace of a single $\mathrm{CdSe} / \mathrm{ZnS}$ nanocrystal on the glass under $488 \mathrm{~nm}$ excitation at room temperature. The on-off PL blinking behavior is clearly observed on the glass substrate. The PL blinking behavior can be analyzed by means of a threshold method, ${ }^{4}$ and the threshold was chosen to divide the on and off states as indicated by a dotted line in Fig. 3(a). The time distribution of the on and off states can be fitted very well by power law functions, $P(t)=A t^{-\alpha}$ [inset of Fig. 3(a).$^{7-11}$ The indices of the power law functions are derived as $1.79 \pm 0.03$ (on-time) and 1.68 \pm 0.02 (off-time), which are consistent with previously reported ones by various groups. ${ }^{8-11,19,20}$ Under the same experimental conditions, we studied the PL intensity time-traces of single nanocrystals on metal surfaces.

Figures 3(b) and 3(c) show the typical PL intensity timetraces of a single $\mathrm{CdSe} / \mathrm{ZnS}$ nanocrystal on the rough and flat $\mathrm{Au}$ surfaces, respectively. These PL blinking behaviors on the rough and flat Au surfaces are completely different from those on the glass surface in Fig. 3(a). The frequency of

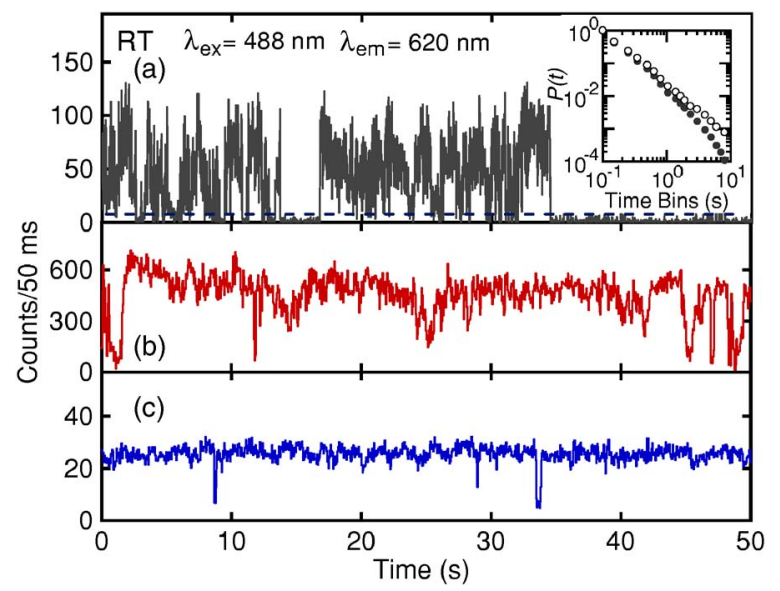

FIG. 3. (Color online) (a)-(c) PL intensity time-traces of single $\mathrm{CdSe} / \mathrm{ZnS}$ nanocrystals on the glass (top panel), the rough Au surface (middle panel), and the flat Au surface (bottom panel). The detector accumulation time of all traces is $50 \mathrm{~ms}$. The inset of (a) is the histogram $p(t)$ of the on-time (solid circles) and off-time (circles) duration of the PL intensity time trace on the glass. The indices of the power law are derived as $1.79 \pm 0.03$ (on-time) and $1.68 \pm 0.02$ (off-time), respectively.

the PL off-time of single CdSe/ZnS nanocrystals is drastically suppressed, and the PL on-time remains for a very long time on both rough and flat Au surfaces. The PL intensity fluctuation on Au surfaces is much smaller than that on the glass surface. In addition, the maximum value of the PL intensity on the rough Au surface ( $\sim 700$ counts/50 ms) increases significantly more than that on the glass surface $(\sim 120$ counts $/ 50 \mathrm{~ms})$. This observation is consistent with the results of the PL image in Fig. 2(a). By contrast, the PL intensity on the flat Au surface decreases significantly.

Here, we discuss the behavior of the PL time-trace both on the rough and flat Au surfaces. It has been accepted that the ionization of nanocrystals under light excitation plays an important role in the PL blinking of semiconductor nanocrystals. ${ }^{9,10}$ The off-time state continues as long as the nanocrystal is ionized. The ionized period is limited by the trapped lifetime of ejected charges in the deep trap states of the glass, ranging from the order of microseconds to minutes. Therefore, we can detect PL blinking on the glass even at room temperature in our observable time scale, as shown in Fig. 3(a). It is considered that the suppression of PL blinking both on the rough and flat Au surfaces is caused by the shortening of the ionized period.

PL decay dynamics was studied under $150 \mathrm{fs}, 490 \mathrm{~nm}$ laser excitation at room temperature using a streak camera. The PL decay curves in Fig. 4 show that the fast-decay PL lifetimes of $\mathrm{CdSe} / \mathrm{ZnS}$ nanocrystals both on the rough and flat $\mathrm{Au}$ surfaces of about $150-200 \mathrm{ps}\left(\tau_{P L \text {,metal }}\right)$ are much shorter than that of about $10 \mathrm{~ns}$ on the glass $\left(\tau_{P L}\right)$. The shorter PL lifetimes on the Au surfaces are governed by the fast energy transfer from the nanocrystals to the Au substrates, and the energy transfer rate on Au surfaces depends scarcely on the surface condition, rough or flat. The photoexcited electrons and holes in nanocrystals escaped into metals or the neutralization of charges in nanocrystals occurs 


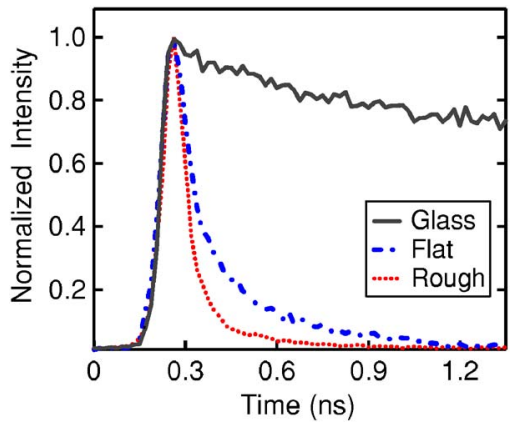

FIG. 4. (Color online) PL decay dynamics of CdSe/ZnS nanocrystals on the glass and the rough and flat Au surfaces in macroscopic ensemble-averaged experiments.

rapidly through the charge injection from the metal surfaces. Fast energy transfer between Au surfaces and nanocrystals drastically shortens the ionized period of the nanocrystals (the lifetime of the ionization state of the nanocrystal), and then the suppression of the PL blinking occurs.

The dynamic behavior of the PL properties of nanocrystals on the rough Au surface is quite similar to that on the flat Au surface, as mentioned above: shortening of the PL lifetime and suppression of PL blinking. Let us consider why the PL enhancement occurs in single CdSe/ZnS nanocrystals on rough $\mathrm{Au}$ surfaces. We have measured the PL intensity of single $\mathrm{CdSe} / \mathrm{ZnS}$ nanocrystals as a function of the excitation light wavelength. In order to evaluate the differences in the PL intensities on the glass and Au surfaces, the PL intensity ratio of a single CdSe/ZnS nanocrystal $\left(I_{\text {gold }} / I_{\text {glass }}\right)$ is introduced, where $I_{\text {gold }}\left(I_{\text {glass }}\right)$ denotes the PL intensities per $50 \mathrm{~ms}$ on the Au surfaces (glass surfaces).

Figure 5 shows the excitation wavelength dependence of the PL intensity ratio $I_{\text {gold }} / I_{\text {glass }}$ on the rough (solid circles) and flat (solid squares) Au surfaces, where $I_{\text {gold }}$ and $I_{\text {glass }}$ are the average PL intensities from the brightest 20 nanocrystals plotted as a function of the excitation wavelength. The PL intensity of a single CdSe/ $\mathrm{ZnS}$ nanocrystal on the rough $\mathrm{Au}$ surface increases about 7 times more at around $490 \mathrm{~nm}$ than

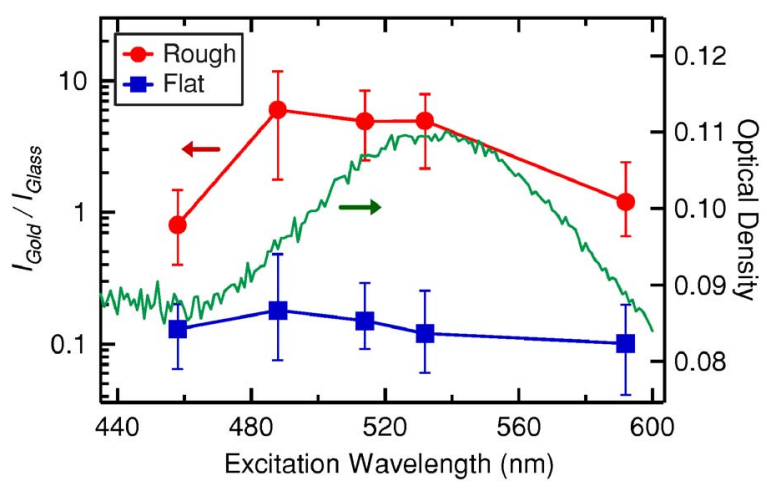

FIG. 5. (Color online) PL intensity ratio $\left(I_{\text {gold }} / I_{\text {glass }}\right)$ as a function of the excitation laser wavelength, where $I_{\text {gold }}$ and $I_{\text {glass }}$ denote the PL intensity of single $\mathrm{CdSe} / \mathrm{ZnS}$ nanocrystals on the $\mathrm{Au}$ and the glass surfaces, respectively. The squares and circles represent the $I_{\text {gold }} / I_{\text {glass }}$ on the flat and rough Au surfaces, respectively. The solid line shows the absorption spectrum of the rough-surface Au film. on the glass surface. On the other hand, the $I_{\text {gold }} / I_{\text {glass }}$ values on the flat Au surface show less than unity in all excitation wavelengths; drastic quenching of the PL intensity occurs on the flat $\mathrm{Au}$ surface. In addition, the optical density of the rough-surface Au film is shown by a solid curve. In the absorption spectrum, the peak located at around $540 \mathrm{~nm}$ indicates the localized plasmon resonance. ${ }^{15,21}$ Thus, the clear resonance behavior of the PL intensity enhancement on the rough $\mathrm{Au}$ surface indicates that the PL intensity enhancement is related to the electric field enhancement due to localized plasmon excitation.

The PL intensity of CdSe/ZnS nanocrystals depends on the absorption, radiative emission, and nonradiative energy dissipation rates in nanocrystal/metal systems. The PL lifetime of the photoexcited nanocrystals on a metal surface, $\tau_{P L \text {,metal }}$, is determined by the radiative and nonradiative recombination rates of nanocrystals, $\gamma_{\text {rad }}$ and $\gamma_{\text {nonrad }}$, and the nonradiative energy transfer rate from nanocrystals to metal surfaces, $\gamma_{\text {metal }} ; 1 / \tau_{P L \text {,metal }}=\gamma_{\text {rad }}+\gamma_{\text {nonrad }}+\gamma_{\text {metal }}$. The PL lifetime on glass is given by $1 / \tau_{P L}=\gamma_{\text {rad }}+\gamma_{\text {nonrad }}$. On both rough and flat Au surfaces, a drastic change in the PL lifetime is clearly observed, and the energy transfer rate from the nanocrystals to the Au surfaces is much larger than the radiative and nonradiative recombination rate of nanocrystals $\left(\gamma_{\text {metal }} \gg \gamma_{\text {rad }}, \gamma_{\text {nonrad }}\right)$. The PL lifetime on metal surfaces is determined by a nonradiative energy transfer rate $\gamma_{\text {metal }}$.

The PL intensity changes of a single $\mathrm{CdSe} / \mathrm{ZnS}$ nanocrystal on the Au surface are mainly determined by two processes: quenching of the excited nanocrystal state due to the energy transfer to metal surface and enhancement of the absorption and radiative emission due to the plasmon-induced electric field. In this case, the PL intensity $\left(I_{\text {gold }}\right)$ is given by, ${ }^{22}$

$$
I_{\text {gold }} \approx \frac{P \gamma_{\text {rad }} I_{\mathrm{abs}}}{\gamma_{\text {metal }}}
$$

where $I_{a b s}$ is the intensity of light absorption and $P$ is the electromagnetic enhancement factor of absorption and radiative emission on the metal surface. The PL intensity ratio $\left(I_{\text {gold }} / I_{\text {glass }}\right)$ in Fig. 5 is proportional to $P \cdot\left(\gamma_{\text {rad }}\right.$ $\left.+\gamma_{\text {nonrad }}\right) / \gamma_{\text {metal }}$. In PL enhancement and quenching, there is no significant difference in the PL lifetime between rough and flat Au surfaces. Moreover, the PL enhancement depends strongly on the excitation light wavelength, and this profile is crudely consistent with the optical absorption spectrum due to the surface plasmon resonance. These observations imply that the enhancement parameter, $P$, is essentially determined by plasmon-induced absorption enhancement rather than radiative emission enhancement. Since the ratio of the decay rates, $\gamma_{\text {metal }}\left(\gamma_{\text {rad }}+\gamma_{\text {nonrad }}\right)$, is about $50(=10 \mathrm{~ns} / 200 \mathrm{ps})$, it is considered that drastic quenching of the PL intensity occurs on the Au surfaces. However, sevenfold PL enhancement is experimentally observed. Therefore, the PL enhancement on rough surfaces is evaluated to be about 300 or more relative to that of the glass surface.

Here, we must consider the difference of the PL intensity time-traces between on the glass and the Au surfaces, as shown in Figs. 2(a)-2(c). From the experimental fact that the 
distribution of the PL blinking probability follows the power law function with an index of $\sim 1.79$, the on-time ratio $R_{\text {on }}$ (the ratio of the "on state" period to the binning time) can be calculated as follows:

$$
R_{o n}=\int_{0}^{\Delta t} t P(t) d t /(\Delta t)
$$

Here, $\Delta t$ is the binning time of $50 \mathrm{~ms}$ in our case, and $P(t)$ is the power law function of the on-time distribution. The ontime ratio $R_{o n}$ is estimated to be about 0.7 on the glass. The suppression of the blinking behavior essentially contributes to the increase in the observed PL intensity. In our experiments, the real enhancement factor $P$ is about $200(\sim 300$ $\times 0.7)$. Both the absorption enhancement by surface-plasmon excitation and the suppression of the PL blinking cause the observed PL enhancement in our experimental conditions. We successfully demonstrated that only single nanocrystal spectroscopy reveals the detailed mechanism of the PL en- hancement and quenching of nanocrystal/metal systems.

In summary, we have studied drastic changes in the PL properties of a single CdSe/ZnS nanocrystal on a Au surface and compared them with those on a glass surface. PL blinking is clearly observed in a single $\mathrm{CdSe} / \mathrm{ZnS}$ nanocrystal on the glass but completely disappears on the flat and rough $\mathrm{Au}$ surfaces. The PL enhancement on rough-surface Au films depends strongly on the excitation wavelength. The PL enhancement on rough Au surfaces is caused by the suppression of PL blinking and electric field enhancement due to localized plasmon excitation.

The authors would like to thank H. Inouye for insightful discussions, and K. Yano, S. Kasai, and T. Ono for the flat Au film preparation and AFM measurements. This work is supported by the Grant-in-Aid for Scientific Research from Japan Society for the Promotion of Science (No. 18340089), the Foundation for C\&C Promotion, and the Research Foundation for Opto-Science and Technology.
*Author to whom correspondence should be addressed. Email address: kanemitu@scl.kyoto-u.ac.jp

${ }^{1}$ S. A. Empedocles, D. J. Norris, and M. G. Bawendi, Phys. Rev. Lett. 77, 3873 (1996).

${ }^{2}$ P. Michler, A. Imamoglu, M. D. Mason, P. J. Carson, G. F. Strouse, and S. K. Buratto, Nature (London) 406, 968 (2000).

${ }^{3}$ M. Nirmal, B. O. Dabbousi, M. G. Bawendi, J. J. Macklin, J. K. Trautman, T. D. Harris, and L. E. Brus, Nature (London) 383, 802 (1996)

${ }^{4}$ K. T. Shimizu, R. G. Neuhauser, C. A. Leatherdale, S. A. Empedocles, W. K. Woo, and M. G. Bawendi, Phys. Rev. B 63, 205316 (2001).

${ }^{5}$ A. L. Efros and M. Rosen, Phys. Rev. Lett. 78, 1110 (1997).

${ }^{6}$ J. Tang and R. A. Marcus, J. Chem. Phys. 123, 204511 (2005).

${ }^{7}$ M. Kuno, D. P. Fromm, A. Gallagher, D. J. Nesbitt, O. I. Micic, and A. J. Nozik, Nano Lett. 1, 557 (2001).

${ }^{8}$ M. Kuno, D. P. Fromm, S. T. Johnson, A. Gallagher, and D. J. Nesbitt, Phys. Rev. B 67, 125304 (2003).

${ }^{9}$ R. Verberk, A. M. van Oijen, and M. Orrit, Phys. Rev. B 66, 233202 (2002).

${ }^{10}$ A. Issac, C. von Borczyskowski, and F. Cichos, Phys. Rev. B 71, 161302(R) (2005).

${ }^{11}$ M. Kuno, D. P. Fromm, H. F. Hamann, A. Gallagher, and D. J.
Nesbitt, J. Chem. Phys. 115, 1028 (2001).

${ }^{12}$ G. Schlegel, J. Bohnenberger, I. Potapova, and A. Mews, Phys. Rev. Lett. 88, 137401 (2002).

${ }^{13}$ R. Verberk and M. Orrit, J. Chem. Phys. 119, 2214 (2003).

${ }^{14}$ K. T. Shimizu, W. K. Woo, B. R. Fisher, H. J. Eisler, and M. G. Bawendi, Phys. Rev. Lett. 89, 117401 (2002).

${ }^{15}$ O. Kulakovich, N. Strekal, A. Yaroshevich, S. Maskevich, S. Gaponenko, I. Nabiev, U. Woggon, and M. Artemyev, Nano Lett. 2, 1449 (2002).

${ }^{16}$ J.-H. Song, T. Atay, S. Shi, H. Urabe, and A. V. Nurmikko, Nano Lett. 5, 1557 (2005).

${ }^{17}$ J. S. Biteen, D. Pacifici, N. S. Lewis, and H. A. Atwater, Nano Lett. 5, 1768 (2005).

${ }^{18}$ J. Gersten and A. Nitzan, J. Chem. Phys. 73, 3023 (1980).

${ }^{19}$ W. G. J. H. M. van Sark, P. L. T. M. Frederix, A. A. Bol, H. C. Gerritsen, and A. Meijerink, ChemPhysChem 3, 871 (2002).

${ }^{20}$ A. Kobitski, C. D. Heyes, and G. U. Nienhaus, Appl. Surf. Sci. 86, 234 (2004).

${ }^{21}$ F. Hache, D. Richard, and C. Flytzanis, J. Opt. Soc. Am. B 3, 1647 (1986).

${ }^{22}$ A. O. Govorov, G. W. Bryant, W. Zhang, T. Skeini, J. Lee, N. A. Kotov, J. M. Slocik, and R. R. Naik, Nano Lett. 6, 984 (2006). 